\title{
Individual Differences in Lexical Repetition Priming
}

\section{The Combined Influence of Language, Mood, and Working Memory Capacity}

\author{
Nikolas Pautz ${ }^{1,2}$ and Kevin Durrheim² \\ 'Department of Psychology, Nottingham Trent University, Nottingham, UK \\ ${ }^{2}$ Discipline of Psychology, School of Applied Human Sciences, University of KwaZulu-Natal, South Africa
}

\begin{abstract}
The current research investigated whether individual differences in working memory capacity (WMC) and affective states have differential effects on lexical-semantic repetition priming outcomes based on whether participants were first- or second-language English speakers. Individual differences in priming effects have often been overlooked in the priming literature. Using logistic mixed-effects models to account for withinsubject variation, the current paper investigated a three-way interaction between WMC, negative affect (NA) score, and language primacy on lexicalsemantic repetition priming outcomes. The results indicate that a statistically significant three-way interaction exists between language primacy, WMC, and NA scores. No significant interaction effect was found for positive affect scores. We present an argument which posits that an individual's primary language and subsequent familiarity with the primed concepts, in conjunction with individual differences in WMC and mood, plays an important role in determining the most effective strategy used to complete a word-stem completion task. The implications of the findings presented highlight that second-language English speakers are more susceptible to priming effects when prime-inducing stimuli are constructed using English lexicon; however, larger WMC and heighted negative affective states help to mitigate these priming effects.
\end{abstract}

Keywords: language, mood, priming, word-stem completion, working memory
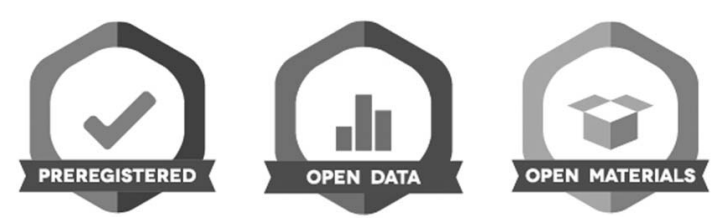

In the current research, we aimed to show how individual differences in mood and working memory capacity (WMC) have conditional differential effects on lexical-semantic priming outcomes based on whether an individual spoke English as a first language (L1) or second language (L2). Language primacy represented an individual's familiarity and fluency with the conceptual structure of the material being used to facilitate a priming effect. We argue that individual differences in L1 and L2 facilitate the use of either heuristic or analytic information-processing strategies when attempting to solve lexical-semantic priming tasks. In addition, we propose that these processing strategies are in turn moderated by both the affective state and WMC of an individual. Elucidating the role of individual differences in priming outcomes is an important endeavor when considering the scrutiny that priming research has received in terms reliability and replicability. What follows is a brief overview of priming effects and the role that language fluency, working memory (WM), and mood may play in determining conditional priming outcomes between L1 and L2 speakers.

\section{Priming}

Priming effects occur when the mere exposure to a stimulus influences an individual's response to a future stimulus. One of the most frequently studied priming effects are those which fall under the category of repetition priming (Soler et al., 2015). Repetition priming generally involves a learner being exposed to a target, such as a word. Once exposure has occurred, Graf and Mandler (1984) suggest that, because prior exposure strengthens the internal schema, the target comes to mind more readily even when only some of its features are presented.

An individual might, for example, study a paragraph that includes a word such as window and is later asked to complete the stem win _ _ with the first word that pops in mind. Individuals who have been exposed to the primed word window have a higher probability of completing the stem with the primed word. Tests which make use of word stems to assess the effect of priming are often called wordstem completion (WSC) tests, which measure lexical- 
semantic priming effects. These tasks are considered to be conceptual tasks in that "they elicit responses based on the retrieval of aspects of stimuli meaning" (Soler et al., 2015, p. 2), as opposed perceptual tasks, which focus on an individual's ability to recall properties of the stimuli presented during encoding. On average, participants have been found to elicit primed responses in around one-fifth of the stems when completing a WSC task (Soler et al., 2015). In contrast, nonprimed individuals complete approximately $4 \%$ of commonly used stems using the primed word (Shaw, 1997).

\section{Language and Conceptual Familiarity}

Research has shown that priming effects, familiarity, and lexical-semantic processing are linked. For example, Dorfman (1994) provided evidence which suggested that priming effects were stronger for items composed of familiar, compared to novel, sublexical elements. Research has also shown that even the most proficient L2 speakers may not always function in their second language as native speakers (McDonough \& Trofimovich, 2011). One factor that has been found to influence priming effects of L2 speakers was L2 vocabulary size: Devitto and Burgess (2004) found that only L2 speakers who had a larger L2 vocabulary displayed semantic priming effects. A possible explanation for this outcome is that priming effects may not occur in individuals who are unfamiliar with the language used to develop the stimuli in priming tasks due to a lack of exposure and subsequent familiarity with the language being tested. Supporting this, Olofsson and Nyberg (1995) found a positive correlation between familiarity and target completion probability in a WSC task. This finding has been reproduced using different semantic priming tasks (Soler et al., 2009). Importantly, Soler et al. (2009) found that less familiar words produce higher priming values. This suggests that L2 speakers who are familiar with the L1 language, but to a lesser extent than L1 speakers (Schmiedtová, 2011), are likely to display greater priming effects than L1 speakers.

The differing levels of conceptual familiarity between L1 and L2 speakers may influence the information-processing strategies used when undertaking an L1-based priming task. As L1 speakers generally have greater familiarity with the language, they may be more likely to rely on heuristic problem-solving strategies to complete the stems, facilitated by the availability heuristic (Ashcraft \& Radvansky, 2010). As L2 speakers with comparatively lower levels of L1 language familiarity display higher priming outcomes (Soler et al., 2009), it is possible that both L1 and L2 speakers use the same heuristic-based strategy to solve the word stem, but it is more effective (resulting in reduced priming effects $^{1}$ ) for those who have greater conceptual familiarity (i.e., L1 speakers). The asymmetrical efficacy of heuristicbased decision-making processes between L1 and L2 speakers may result in differing thresholds that facilitate changes in the strategies used to complete priming tasks, such as more analytical strategies (Pretz et al., 2010). In the current paper, we investigate two cognitive factors which can vary by individual that may trigger a change in the strategies (i.e., heuristic or analytical) used to solve word stems, WMC and mood.

\section{Working Memory Capacity}

$\mathrm{WM}$ is required to process new information while accessing and maintaining old information in long-term memory. Under the WM model proposed by Baddeley and Hitch (1974), the central executive control system has domain general components that include updating information as new stimuli appear, shifting between tasks, and, importantly, inhibiting habitual (i.e., heuristic) responses (Corbin et al., 2010; Mitchell et al., 2002). Individual differences in WMC reflect the limited capacity of an individual's WM.

There is evidence suggesting that WMC and priming effects may be functionally related. Research has shown that priming effects disappear under high WM load (Kiefer et al., 2005; Szuhany et al., 2018). Kiefer et al. (2005) found a negative association between verbal WMC and priming, and Szuhany et al. (2018) reported that priming effects facilitating reward delay were only effective in individuals with lower WMC. Ortells et al. (2017) argued that differential availability of cognitive resources such as WM can reliably influence priming effects at a semantic level of representation while Caggiano et al. (2006) found that active maintenance of primes in WM may suppress more implicit processes, such as heuristics (Whitney et al., 2008). The current paper extends this line of research by focusing on lexical-semantic priming effects as well as the interplay between individual differences in WMC and mood.

\section{Mood}

It has long been thought that an individual's affective state has an influence on their cognitive thought processes and

\footnotetext{
In WSC tasks, participants are generally explicitly instructed to not complete a word stem with a word they had been exposed to in the early stages of the task.
} 
subsequent information processing. Under the dualsystem accounts of cognition, it is hypothesized that positive affect (PA) facilitates heuristic and relational processing, while negative affect (NA) facilitates systematic and item-specific processing (Fiedler et al., 2001; Pretz et al., 2010). The former involves processing incoming information in relation to concepts already formed in memory and drawing connections between these concepts, while the latter involves the processing of unique details and features of stimuli as opposed to considering the stimuli in relation to other already formed concepts (Fiedler et al., 2001). Indeed, positive mood has been shown to increase heuristic-based behavior such as stereotyping (Park \& Banaji, 2000), while NA has been shown to stimulate systematic analytical processing (Bless et al., 1990). Positive mood has been found to impair certain analytically based cognitive functions, such as assessing, planning, and reasoning because it triggers heuristic thinking (Spies et al., 1996).

The influence of mood on WM is relatively well established. Raczy and Orzechowski (2019) suggest that affect induces different types of information processing within WM. Martin and Kerns (2011) found that a positive mood impairs an individual's WMC, arguing that a positive mood increases the spread of activation of items in WM; the increasing spread of items in WM makes it more difficult to retrieve stored concepts. In this paper, we investigated whether the mood of an individual plays a role in predicting lexical priming outcomes between L1 and L2 speakers while considering individual differences in WMC.

\section{The Current Study}

The current research aimed to investigate a three-way interaction between language primacy, $\mathrm{WM}$, and mood on lexical repetition priming effects. Based on previous findings (Devitto \& Burgess, 2004; Soler et al., 2009), we argued that L1 and L2 English speakers have different levels of familiarity with lexical English stimuli and that these different levels of familiarity are likely to play a role in the efficacy of familiarity-based heuristics. Specifically, we argue that L1 speakers may display weaker priming effects in a WSC task because they are able to use heuristics more effectively due to increased levels of exposure (and thus familiarity) with English lexicon (Schmiedtová, 2011), while L2 speakers would display comparatively stronger priming effects due to less efficient heuristic processing due to lower levels of conceptual familiarity with L1 lexicon. Based on prior research suggesting that priming effects are stronger when WMC is low and weaker when WMC is high (Kiefer et al., 2005), we expected to find a differential effect on priming outcomes based on individual differences in WMC and on whether participants are L1 or L2 English speakers. Importantly, we hypothesized that the influence of WMC on the relationship between language primacy and priming effects would be moderated by the affective state of the individual. Specifically, we hypothesized that positive mood facilitates heuristic processing (Park \& Banaji, 2000; Pretz et al., 2010), leading to reduced priming effects when individuals are familiar with the lexical concepts (L1 speakers), but that PA would have a negligible effect on the relationship between priming effects and WMC for L2 speakers due to reduced heuristic efficacy. In contrast, we hypothesize that negative mood would facilitate more analytical processing (Bless et al., 1990; Fiedler et al., 2001), diminishing priming effects on the same task for individuals who are less familiar with the concepts (L2 speakers) and have higher WM capacities, but these conditions may have a detrimental effect on the efficacy of heuristic processing for L1 speakers and induce stronger priming effects.

\section{Materials and Method}

\section{Participants and Screening}

A total of $N=168$ South African university students completed this experiment. Participants were recruited via convenience sampling and were compensated R50 ( $\$ 4.5$ at the time) for their time. Due to data corruption, seven participants were removed. The remaining data were further screened to ensure that the outcomes of the WSC were sound. Overall, 3,357 stems were completed; of these, 456 stems which were either nonresponses or responses which were above or below six characters long were removed. Additionally, 139 stems were removed for being nonsense words and 37 stems were removed for being proper nouns. After this, any participants who had not completed at least half of the stems were removed, leaving $N=155$ participants and 2,725 stems.

The remaining participants had a mean age of 23.61 $( \pm 4.8) ; N=100$ were female, and $N=55$ were male. $N=57$ participants self-selected English as their L1 language, while $N=98$ participants self-selected English as their L2 language.

Despite the asymmetric size between the two speaker groups, the characteristics of the distributions were largely similar. Two-sample Kolmogorov-Smirnov tests showed that no significant differences in distributions were found between L1 and L2 speakers for both NA $(D=0.06$, $p=.99)$ and PA $(D=.12, p=.67)$ scores; however, the WMC scores did have substantially different distributions $(D=.31, p<.05)$ between the speaker groups. 


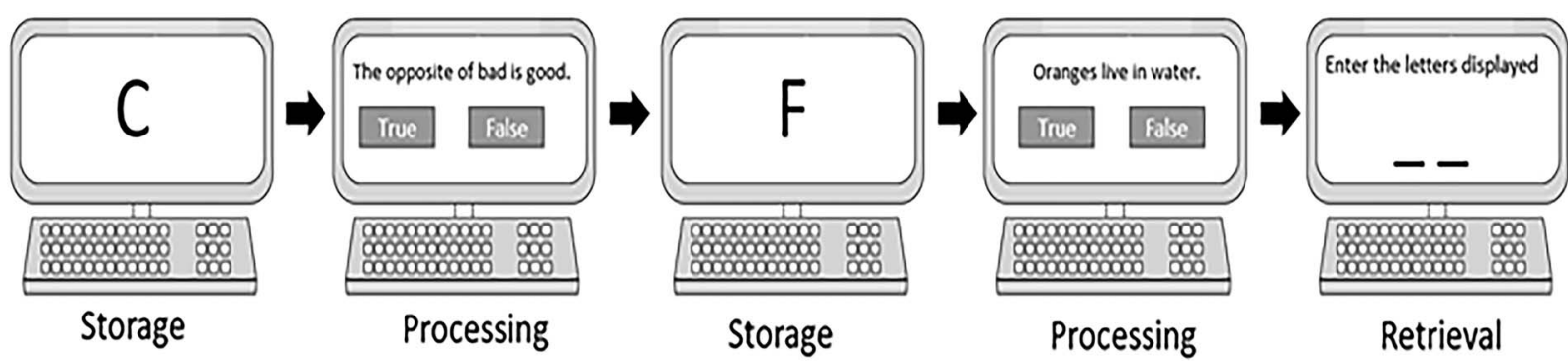

Figure 1. A schematic illustration of the stages involved in the WMC task.

\section{Affect Manipulation and Stimuli}

Participants were allocated quasirandomly into one of two experimental groups: PA $(N=72)$ and NA $(N=83)$. Within the PA group, there were $N=26 \mathrm{~L} 1$ and $N=46 \mathrm{~L} 2$ speakers; within the NA group, there were $N=31 \mathrm{~L} 1$ speakers and $N=52 \mathrm{~L} 2$ speakers. To induce and measure the affective state of each participant, two steps were performed. First, the participants in the PA group were asked to watch a movie-like slide show that contained 75 pictures within the positive category of the Geneva Affective Picture Database (GAPED; Dan-Glauser \& Scherer, 2011). Each picture was displayed for 5 seconds before moving onto the next picture in the sequence. Participants in the NA group were similarly asked to watch a slide show comprising 75 pictures from the "human concerns" category of the "negative affect" category in the GAPED. The order of the presentation was randomized. The average valence for the positive group was $89.65(S D=6.2)$, and the human concerns category of the NA group was $27.95(S D=12.4$; Dan-Glauser \& Scherer, 2011). Directly after viewing the stimuli, participants completed the PANAS (Watson et al., $1988)$ to quantize their affective state. Both the PA subscale $(\alpha=.86[.83 ; .89])$ and the NA subscale $(\alpha=.87[.84$; .9]) were reliable. A limitation of the study design is that the PANAS was only administered after, and not also before, affect manipulation.

\section{Repetition Priming Stimuli}

A WSC task was used to measure the effects of lexical repetition priming. This WSC task had two blocks; each block contained a novel L1 paragraph which participants were asked to read through once. Each paragraph contained 12 six-letter words which were used as three-letter word stems that were noncompatible with the other block (see Table $1 \mathrm{~A}$ in the Appendix for an overview of the stems, words, and frequencies). All words in the paragraphs were presented using the same font and size. After reading the paragraph and then completing a 5-minute filler task, participants proceeded to the next stage of the study. In this stage, they were asked to complete the six-lettered stems (with the three missing letters represented by underscores) in an unspeeded manner with explicit instructions not to use a word that was included in the associated paragraph.

To determine reliability, the level of priming for each participant was measured by calculating the proportion of primed word responses. The consistency of the mean proportion of primed responses between the two blocks was acceptable $(r=.64[.52, .72])$. When modeling the priming outcomes, each stem was treated as a binary outcome (primed or nonprimed response).

\section{Working Memory Capacity}

To measure verbal WM, participants were asked to complete a computerized Reading Span task based on procedures outlined by Unsworth et al. (2009). Participants were shown an element which they needed to store (a letter ranging from $\mathrm{A}$ to $\mathrm{Z}$ ) on the screen for $1,000 \mathrm{~ms}$. After viewing a single element, participants were then shown a statement (e.g., Dogs are known to lay eggs) which they were required to process (identify if the statement was true or false). After processing, another element was presented, with a minimum of two and a maximum of seven presented in each trial. When the participants had viewed all the elements and processed all statements in a trial, a box with letters appeared on the screen (always including the elements shown in the trial, but also including elements that were not). Scoring was done based on a threshold of $4 / 5$ trials per element size. For instance, if a participant had $4 / 5$ five-element trials completed successfully, but $3 / 5$ trials

Table 1. Descriptive statistics for PANAS scores between the positive and negative experimental groups

\begin{tabular}{llll}
\hline & Affect group & \multicolumn{1}{c}{$M( \pm S D)$} & $95 \% \mathrm{Cl}[\mathrm{L}, \mathrm{U}]$ \\
\hline PA score & NA group & $23.4( \pm 7.39)$ & {$[21.8,25]$} \\
& PA group & $29.4( \pm 11.2)$ & {$[26.9,32]$} \\
NA score & NA group & $25.5( \pm 9.47)$ & {$[23.4,27.5]$} \\
& PA group & $12.9( \pm 4.89)$ & {$[11.7,14]$} \\
\hline
\end{tabular}

Note. $N A=$ negative affect; $P A=$ positive affect. 
completed successfully for six-element trials, they were assigned a span score of 5 . This method of scoring should be viewed as a conservative measure of WMC. An example schematic of the WMC task is shown in Figure 1.

\section{Results}

It was important to determine if the affect manipulation was effective (the descriptive statistics of the affect scores between the two experimental affect groups are displayed in Table 1). Using a multivariate ANOVA with PA and NA scores as the DVs and Affect Group as the fixed factor, the results indicated that there was a significant main effect of Affect Group for both positive $\left(F(1,153)=16.19, p<.001, \eta_{\mathrm{p}}{ }^{2}=.096\right)$ and negative $\left(F(1,153)=103.37, p<.001, \eta_{\mathrm{p}}{ }^{2}=.4\right)$. The descriptive statistics in Table 1 suggest that the mood manipulation was less effective at reducing PA (a mean change of six between affect groups) despite effectively stimulating NA (a mean change of 12.6 between affect groups).

L1 English speakers had lower average primed response proportions $(M=0.25, \pm 0.43)$ than L2 speakers $(M=0.32$, \pm 0.46 ). A logistic mixed-effects model using the lme 4 package in $\mathrm{R}$ was computed to examine the effects of $\mathrm{WMC}$ and affect on these language differences. This was appropriate as the inclusion of participants as random slopes $(D=3,151)$ significantly improved the fit when compared to an intercept-only model $(D=3,294)$,
$\mathrm{X}^{2}{ }_{(1)}=-142.61, p<.001$. Additionally, using the Modified Moment Estimate with Weights Proportional to Cluster Size method in the iccbin $\mathrm{R}$ package alongside the Fleiss-Cuzick method of calculating the $95 \%$ CI, the ICC $(.15[.04, .19])$ was significant and warranted the use of mixed-effects models.

Following this, affect scores were logarithmically transformed to aid model convergence and normalize the distribution of scores. Table 2 illustrates the outcomes of the two primary models. Model 1 includes PA scores both as an individual predictor and an interaction between WMC and Language; Model 2 excludes these predictors as they were not found to be statistically significant and reduced the overall fit of the model.

The outcomes of Model 2 suggest that the interaction of language, WMC, and NA has a statistically significant conditional relationship on semantic priming outcomes. Figure 2 illustrates the nature of this conditional relationship (see Figure A1 in the Appendix for an illustration of the interaction with PA scores).

Inferring from Model 2 and the illustration of the interaction (Figure 2), there were observable differences between L1 and L2 speakers in the direction of the relationship between the probability of a participant having a primed response and individual WMC, and NA scores. L1 speakers were less likely to respond with a primed answer if they had lower WMC and had a lower NA score. L1 participants with higher WMC showed a linear increase in probability of primed response, with those with higher NA scores having the

Table 2. Logistic mixed-effects regression models with binary priming outcomes ( $1=$ primed response; $0=$ nonprimed response) as the dependent variable

\begin{tabular}{|c|c|c|c|c|c|}
\hline & Estimate & $95 \% \mathrm{Cl}[\mathrm{L}, \mathrm{U}]$ & SE & $z$-value & p \\
\hline Language (1 = L1; 2 = L2) & 1.269 & {$[0.31,2.27]$} & 0.506 & 2.506 & .012 \\
\hline WMC & 0.260 & {$[-0.04,0.59]$} & 0.165 & 1.579 & .114 \\
\hline NA score (log) & 1.392 & {$[1.22,5.08]$} & 0.452 & 3.082 & .002 \\
\hline PA score (log) & -0.364 & {$[-2.51,0.86]$} & 0.402 & -0.905 & .366 \\
\hline Affect group (1 = NAG; 2 = PAG) & 0.747 & {$[0.34,1.14]$} & 0.210 & 3.558 & $<.001$ \\
\hline Language $\times \mathrm{WMC} \times \mathrm{NA}$ & -0.120 & {$[-0.51,-0.03]$} & 0.056 & -2.145 & .032 \\
\hline Language $\times \mathrm{WMC} \times \mathrm{PA}$ & 0.047 & {$[-0.1,0.32]$} & 0.051 & 0.924 & .356 \\
\hline \multicolumn{6}{|c|}{ Model 2 (excluding PA scores) AIC = 3,141; BIC = 3,183 } \\
\hline & Estimate & $95 \% \mathrm{Cl}[\mathrm{L}, \mathrm{U}]$ & SE & z-value & $p$ \\
\hline Language $(1=\mathrm{L} 1 ; 2=\mathrm{L} 2)$ & 1.076 & {$[1.14,3.82]$} & 0.304 & 3.542 & $<.001$ \\
\hline WMC & 0.309 & {$[0.01,0.61]$} & 0.154 & 2.003 & .045 \\
\hline NA score (log) & 1.424 & {$[0.53,2.32]$} & 0.473 & 3.013 & .003 \\
\hline Affect group (1 = PAG; 2 = NAG) & 0.742 & {$[0.35,1.14]$} & 0.199 & 3.729 & $<.001$ \\
\hline Language $\times \mathrm{WMC} \times \mathrm{NA}$ & -0.079 & {$[-0.34,-0.03]$} & 0.034 & -2.298 & .022 \\
\hline
\end{tabular}

Note. AIC = Akaike information criterion; $\mathrm{BIC}=$ Bayesian information criterion; NA = negative affect PANAS score; NAG = NA group; PA = positive affect PANAS score; PAG = PA group; $W M C=$ working memory capacity .

$95 \%$ Cls were calculated using the bootstrap method with 1,000 simulations. Bolded entries represent statistically significant effects using an alpha of 0.05 . 


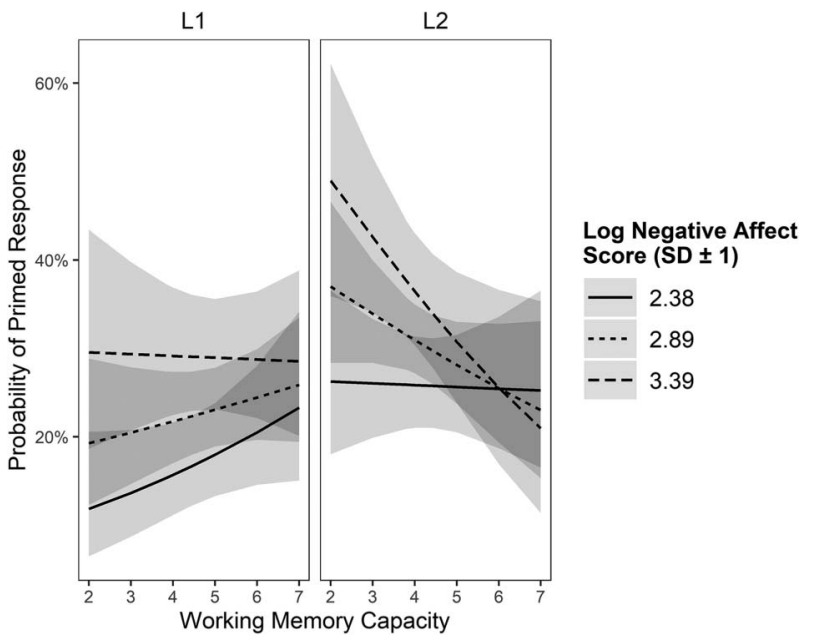

Figure 2. An illustration of the three-way interaction between language, WMC, and NA score on lexical repetition priming outcomes shown in Table 2 (Model 2). Shaded areas represent the 95\% Cl. $N A$ = negative affect; $W M C$ = working memory capacity.

highest likelihood of responding with a primed word. In contrast, L2 speakers showed a markedly different pattern; individuals with a lower WMC were more likely to have responded with a primed word, but individuals with a higher WMC were substantially less likely to so, with individuals who had a high WMC and a high NA score being the least likely of all L2 speakers to respond with a primed answer. This contrasts with L1 speakers where individuals with low WMC and NA were the least likely to respond with a primed word.

\section{Discussion}

The present research investigated if individual differences in WMC, moderated by mood, predicted lexical-semantic priming outcomes differentially based on whether participants were L1 or L2 English speakers. The foundation of the working hypothesis that such conditional effects would be found was based on the idea that L1 and L2 speakers have different levels of familiarity of lexical concepts of the L1 language on which the WSC was based (Devitto \& Burgess, 2004; McDonough \& Trofimovich, 2011). These differing levels of familiarity asymmetrically facilitate the efficacy of heuristic information and problem-solving strategies: We hypothesized that L1 speakers would be able to use heuristic strategies more effectively via the utilization of the familiarity heuristic, while L2 speakers would use heuristic strategies less effectively as their conceptual familiarity with the L1 lexicon was not as deep as L1 speakers (McDonough \& Trofimovich, 2011; Stoffelsma, 2019), resulting in increased priming effects. Furthermore, we hypothesized that the heuristic strategies used by L1 speakers to complete lexical-priming tasks would be more effective when WMC was lower and positive affective scores were higher, while the heuristic strategies used by L2 speakers would be less effective under these same conditions, but more effective when heuristic strategies were replaced by analytical ones when WMC and NA scores were higher (Corbin et al., 2010; Pretz et al., 2010).

The results presented largely support the primary hypothesis that language primacy, WM, and mood interact and have conditional effects on lexical-semantic repetition priming outcomes. The finding that L1 speakers had lower probabilities to respond with primed words when negative mood and individual WMC were low supports the idea that heuristic strategies are being used - higher WMC has been found to suppress habitual and heuristic responses (Corbin et al., 2010; Mitchell et al., 2002) - and that these L1 heuristic strategies are effective at minimizing priming effects. The relationship between WMC and priming effects for L1 speakers was further moderated by NA score; the finding that L1 participants with lower NA were the least likely out of all L1 participants to respond with a primed word inversely supports the idea that higher levels of NA facilitate systematic and analytical strategies (Fiedler et al., 2001; Pretz et al., 2010). Overall, these outcomes support the idea that the deeper conceptual familiarity of L1 lexicon afforded to L1 English speakers facilitates a highly effective heuristic strategy to complete lexical-semantic priming tasks such as the WSC. The efficacy of the strategy is moderated jointly by individual differences in WMC and mood.

The finding that PA did not moderate the effects of WMC and language primacy was counterintuitive to what was expected. As PA has been argued to facilitate heuristic processing (Park \& Banaji, 2000) akin to how NA has been posited to facilitate more analytical processing strategies (Pretz et al., 2010), we expected higher levels of PA would facilitate heuristic processing in L1 when WMC was low. This finding hints that it is not necessarily the stimulation of PA that facilitates heuristic processing used in lexical repetition priming tasks, but possibly the suppression of NA.

The influence of WMC and NA score in L2 speakers stood in almost direct contrast to the patterns found in L1 speakers, supporting the hypothesis that individual differences in WMC and mood have differential effects on L1 lexical-semantic priming tasks depending on whether subjects are L1 or L2 speakers. When WMC scores were low, the pattern of priming probabilities was similar between the different levels of NA for both L1 and L2 speakers, although L2 speakers had higher overall estimates of priming outcomes, which may be due to lower levels of conceptual familiarity. Under these low WMC conditions, individuals who had lower levels of NA were the least likely to respond with a primed answer. This reinforces the idea that low WMC and low NA promote a cognitive environment where heuristic strategies are favored when completing repetition priming tasks and that 
these strategies are more effective in L1 speakers. This interpretation is supported by existing research which reports that priming effects disappear when WMC is high (Kiefer et al., 2005) and are evident when WMC is low (Szuhany et al., 2018).

Expanding on this is that, in contrast to L1 speakers, as WMC and NA scores in the L2 group increased, the probability of responding with a primed word decreased substantially. This is likely due to increases in both WMC suppressing habitual responses (Corbin et al., 2010; Mitchell et al., 2002) and NA stimulating more analytical strategies (Pretz et al., 2010). Both cognitive elements are important for individuals to switch from heuristic strategies to analytical and systematic alternatives: L2 participants who had lower levels of NA displayed only a marginally reduced probability with as WMC increased. This suggests that it is both the suppression of habitual responses and the facilitation of analytical processing strategies, which triggers the threshold required for $\mathrm{L} 2$ participants to move away from heuristic strategies toward more systematic ones when completing a lexical repetition task.

It is important to acknowledge that optimal conditions for L1 heuristic processing still appear to result in lower probabilities of primed responses than optimal conditions for L2 systematic processing when completing a lexical-semantic repetition priming task. This suggests that $\mathrm{L} 1$ speakers have, to some extent, an inherent heuristic advantage when priming effects are reliant on familiarity and fluency with the conceptual representations. While L2 speakers are able to compensate for their disadvantage - to a substantial degree - by utilizing more analytical problem-solving strategies when cognitive prerequisites such as WMC and mood levels have been met, $\mathrm{L} 2$ speakers maintain increased susceptibility to priming effect. Future research with a focus on qualitative levels of familiarity for the L1 language and lexical concepts within a semantic-based repetition priming task would be beneficial in supporting the findings presented. Finally, future research might consider an experimental approach as opposed to the individual differences approach taken in the current research. Careful manipulation of WMC, particularly for lower capacities, may provide additional insight into the complex relationship that exists between language primacy, affect, and WM.

\section{References}

Ashcraft, M. H., \& Radvansky, G. A. (2010). Chapter 3: Perception and pattern recognition. Cognition, 5, 65-110.

Baddeley, A. D., \& Hitch, G. (1974). Working memory. In G. H. Bower (Ed.), Psychology of learning and motivation (Vol. 8, pp. 47-89). Academic Press. https://doi.org/10.1016/S0079-7421(08)60452-1
Bless, H., Bohner, G., Schwarz, N., \& Strack, F. (1990). Mood and persuasion. Personality and Social Psychology Bulletin, 16(2), 331-345. https://doi.org/10.1177/0146167290162013

Caggiano, D., Jiang, Y., \& Parasuraman, R. (2006). Aging and repetition priming for targets and distracters in a working memory task. Aging, Neuropsychology, and Cognition, 13(3-4), 552-573. https://doi.org/10.1080/138255890969555

Corbin, J., McElroy, T., \& Black, C. (2010). Memory reflected in our decisions: Higher working memory capacity predicts greater bias in risky choice. Judgment and Decision Making, 5(2), 110.

Dan-Glauser, E. S., \& Scherer, K. R. (2011). The Geneva affective picture database (GAPED): A new 730-picture database focusing on valence and normative significance. Behavior Research Methods, 43(2), 468-477. https://doi.org/10.3758/s13428-011-0064-1

Devitto, Z., \& Burgess, C. (2004). Theoretical and methodological implications of language experience and vocabulary skill: Priming of strongly and weakly associated words. Brain and Cognition, 55(2), 295-299. https://doi.org/10.1016/j.bandc.2004.02.018

Dorfman, J. (1994). Sublexical components in implicit memory for novel words. Journal of Experimental Psychology: Learning, Memory, and Cognition, 20(5), 1108-1125. https://doi.org/10.1037/0278-7393.20.5.1108

Fiedler, K., Nickel, S., Muehlfriedel, T., \& Unkelbach, C. (2001). Is mood congruency an effect of genuine memory or response bias? Journal of Experimental Social Psychology, 37(3), 201-214. https://doi.org/10.1006/jesp.2000.1442

Graf, P., \& Mandler, G. (1984). Activation makes words more accessible, but not necessarily more retrievable. Journal of Verbal Learning and Verbal Behavior, 23(5), 553-568. https://doi.org/10. 1016/S0022-5371(84)90346-3

Kiefer, M., Ahlegian, M., \& Spitzer, M. (2005). Working memory capacity, indirect semantic priming, and stroop interference: Pattern of interindividual prefrontal performance differences in healthy volunteers. Neuropsychology, 19(3), 332-344. https:// doi.org/10.1037/0894-4105.19.3.332

Martin, E. A., \& Kerns, J. G. (2011). The influence of positive mood on different aspects of cognitive control. Cognition \& Emotion, 25(2), 265-279. https://doi.org/10.1080/02699931.2010.491652

McDonough, K., \& Trofimovich, P. (2011). Using priming methods in second language research. Taylor \& Francis.

Mitchell, J. P., Macrae, C. N., \& Gilchrist, I. D. (2002). Working memory and the suppression of reflexive saccades. Journal of Cognitive Neuroscience, 14(1), 95-103. https://doi.org/10.1162/ 089892902317205357

Olofsson, U., \& Nyberg, L. (1995). Determinants of word fragment completion. Scandinavian Journal of Psychology, 36(1), 59-64. https://doi.org/10.1111/j.1467-9450.1995.tb00968.x

Ortells, J. J., Álvarez, D., Noguera, C., Carmona, E., \& de Fockert, J. W. (2017). The influence of working memory load on expectancybased strategic processes in the stroop-priming task. Frontiers in Psychology, 08, 129 https://doi.org/10.3389/fpsyg.2017.00129

Park, J., \& Banaji, M. R. (2000). Mood and heuristics: The influence of happy and sad states on sensitivity and bias in stereotyping. Journal of Personality and Social Psychology, 78(6), 1005-1023. https://doi.org/10.1037/0022-3514.78.6.1005

Pretz, J. E., Totz, K. S., \& Kaufman, S. B. (2010). The effects of mood, cognitive style, and cognitive ability on implicit learning. Learning and Individual Differences, 20(3), 215-219. https://doi.org/10. 1016/j.lindif.2009.12.003

Raczzy, K., \& Orzechowski, J. (2021). When working memory is in a mood: Combined effects of induced affect and processing of emotional words. Current Psychology, 40(6), 2843-2852. https:// doi.org/10.1007/s12144-019-00208-x

Schmiedtová, B. (2011). Do L2 speakers think in the L1 when speaking in the L2? Vigo International Journal of Applied Linguistics, 8(1), 138-179.

Shaw, R. J. (1997). Unprimed stem completion is only moderately predicted by word frequency and length. Behavior Research 
Methods, Instruments, \& Computers, 29(3), 401-424. https://doi. org/10.3758/BF03200594

Soler, M. J., Dasí, C., \& Ruiz, J. C. (2009). Datos normativos de 269 fragmentos de palabras españolas a partir de la base de Dasí, Soler y Ruiz (2004) [Normative data of 269 Spanish word fragments from the extension of Dasí, Soler and Ruiz (2004) database]. Psicológica, 30(1), 91-117.

Soler, M. J., Dasí, C., \& Ruiz, J. C. (2015). Priming in word stem completion tasks: Comparison with previous results in word fragment completion tasks. Frontiers in Psychology, 6, 172. https://doi.org/10.3389/fpsyg.2015.01172

Spies, K., Hesse, F., \& Hummitzsch, C. (1996). Mood and capacity in Baddeley's model of human memory. Zeitschrift Für Psychologie Mit Zeitschrift Für Angewandte Psychologie, 204(4), 367-381.

Stoffelsma, L. (2019). English vocabulary exposure in South African township schools: Pitfalls and opportunities. Reading \& Writing, 10(1), 1-10. https://doi.org/10.4102/rw.v10i1.209

Szuhany, K. L., MacKenzie, D., Jr., \& Otto, M. W. (2018). The impact of depressed mood, working memory capacity, and priming on delay discounting. Journal of Behavior Therapy and Experimental Psychiatry, 60, 37-41. https://doi.org/10.1016/j.jbtep.2018.03.001

Unsworth, N., Brewer, G. A., \& Spillers, G. J. (2009). There's more to the working memory capacity-fluid intelligence relationship than just secondary memory. Psychonomic Bulletin \& Review, 16(5), 931-937. https://doi.org/10.3758/PBR.16.5.931

Van Heuven, W. J., Mandera, P., Keuleers, E., \& Brysbaert, M. (2014). SUBTLEX-UK: A new and improved word frequency database for British English. Quarterly Journal of Experimental Psychology, 67(6), 1176-1190. https://doi.org/10.1080\%2F17470218.2013.850521

Watson, D., Clark, L. A., \& Tellegen, A. (1988). Development and validation of brief measures of positive and negative affect: The PANAS scales. Journal of Personality and Social Psychology, 54(6), 1063-1070. https://doi.org/10.1037/0022-3514.54.6.1063

Whitney, P., Rinehart, C. A., \& Hinson, J. M. (2008). Framing effects under cognitive load: The role of working memory in risky decisions. Psychonomic Bulletin \& Review, 15(6), 1179-1184. https://doi.org/10.3758/PBR.15.6.1179

\section{History}

Received October 21, 2020

Revision received September 2, 2021

Accepted September 3, 2021

Published online December 17, 2021

\section{Acknowledgments}

Thank you to Oana Catinas for her assistance with data collection.

\section{Open Data}

A pre-print was archived on a public forum https://psyarxiv.com/ $7 \mathrm{dy} 8 \mathrm{~m} /$. The data that support the findings of this study are openly available in The Center for Open Science (OSF) at http://doi.org/ 10.17605/OSF.IO/UQVFE. The materials used to collect the data can be viewed and downloaded at https://app.gorilla.sc/openmaterials/238332.

\section{Funding}

This research was supported by the National Research Foundation's Innovation and Scarce Skills Development Doctoral scholarship (SFH150624120525).

\section{ORCID}

Nikolas Pautz

(iD) https://orcid.org/0000-0002-5366-5925

\section{Nikolas Pautz}

Department of Psychology

Nottingham Trent University

Taylor Building

5LP Chaucer St

Nottingham, Nottinghamshire NG1 4FQ

United Kingdom

nikolas.pautz@ntu.ac.uk 


\section{Appendix}

Table A1. List of stems and words for the word-stem completion task

\begin{tabular}{|c|c|c|c|}
\hline Order & Word stem & Word & Log Zipf frequency \\
\hline 1 & STA & Stands & 4.64 \\
\hline 2 & WAT & Waters & 4.34 \\
\hline 3 & FRI & Friend & 5.29 \\
\hline 4 & RET & Retire & 4.38 \\
\hline 5 & REG & Region & 4.79 \\
\hline 6 & JOY & Joyful & 3.27 \\
\hline 7 & MON & Months & 5.36 \\
\hline 8 & AMO & Amount & 5.07 \\
\hline 9 & RAT & Rather & 5.39 \\
\hline 10 & BUS & Bustle & 3.28 \\
\hline 11 & LIV & Living & 5.31 \\
\hline 12 & BET & Better & 5.76 \\
\hline 13 & CRE & Create & 4.95 \\
\hline 14 & STA & Starry & 3.22 \\
\hline 15 & LON & Lonely & 4.35 \\
\hline 16 & STU & Sturdy & 3.42 \\
\hline 17 & VIS & Vision & 4.56 \\
\hline 18 & PAN & Panels & 3.90 \\
\hline 19 & SCR & Screen & 4.61 \\
\hline 20 & WIN & Winked & 2.67 \\
\hline 21 & SMI & Smiled & 3.54 \\
\hline 22 & WAN & Wanted & 5.56 \\
\hline 23 & BRE & Breeze & 4.19 \\
\hline 24 & PAS & Passed & 4.77 \\
\hline
\end{tabular}

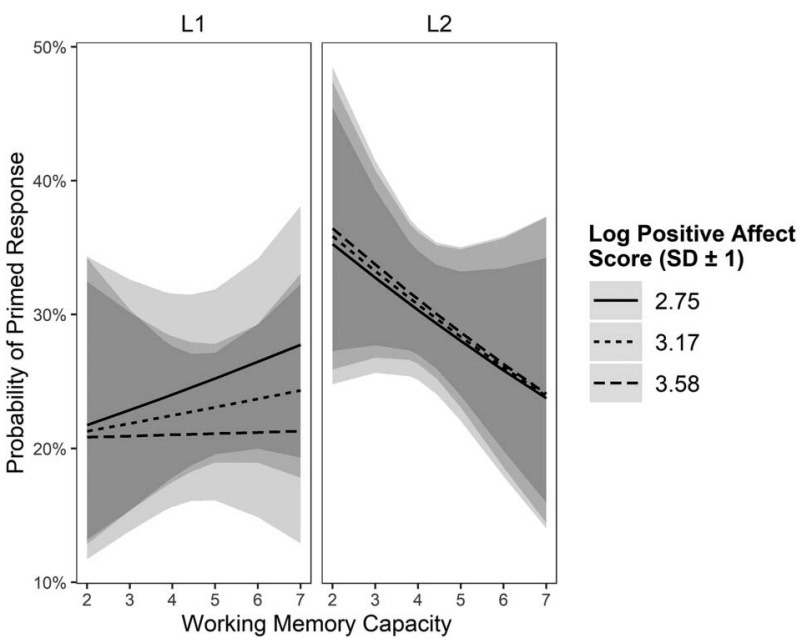

Figure A1. An illustration of the three-way interaction between language, WMC, and PA score on lexical repetition priming outcomes shown in Table 2 (Model 2). Shaded areas represent the 95\% $\mathrm{Cl}$. PA = positive affect; $W M C$ = working memory capacity

Note. The Zipf frequency is a logarithmic scale and goes roughly from 1 (verylow-frequency words) to 6 (very-high-frequency words). These frequencies were taken from the Subtlex-UK database (van Heuven et al., 2014). 